\title{
AN INVERSE EIGENVALUE PROBLEM FOR AN ARBITRARY MULTIPLY CONNECTED BOUNDED REGION: AN EXTENSION TO HIGHER DIMENSIONS
}

\author{
E.M.E. ZAYED \\ Mathematics Department, Faculty of Science \\ Zagazig University, Zagasiz, Egypt \\ (Received August 30, 1991 and in revised form September 3, 1992)
}

\begin{abstract}
The basic problem in this paper is that of determining the geometry of an arbitrary multiply connected bounded region in $R^{3}$ together with the mixed boundary conditions, from the complete knowledge of the eigenvalues $\left\{\lambda_{\jmath}\right\}_{j=1}^{\infty}$ for the negative Laplacian, using the asymptotic expansion of the spectral function $\Theta(t)=\sum_{j=1}^{\infty} \exp \left(-t \lambda_{\jmath}\right)$ as $t \rightarrow 0$.
\end{abstract}

KEY WORDS AND PHRASES. Inverse problem, Laplace's operator, eigenvalue problem and spectral function.

1991 AMS SUBJECT CLASSIFICATION CODES. 35K, 35P.

\section{INTRODUCTION.}

The underlying problem is to deduce the precise shape of a membrane from the complete knowledge of the eigenvalues $\{\lambda,\}_{j=1}^{\infty}$ for the negative Laplacian $-\Delta_{3}=-\sum_{i=1}^{3}\left(\frac{\partial}{\partial x^{2}}\right)^{2}$ in the $\left(x^{1}, x^{2}, x^{3}\right)$ - space.

Let $\Omega \subseteq R^{3}$ be a simply connected bounded domain with a smooth bounding surface $S$. Consider the Dirichlet/Neumann problem

$$
\begin{aligned}
& \left(\Delta_{3}+\lambda\right) u=0 \text { in } \Omega, \\
& u=0 \text { or } \frac{\partial u}{\partial n}=0 \text { on } S,
\end{aligned}
$$

where $\frac{\partial}{\partial n}$ denotes differentiation along the inward pointing normal to $S$. Denote its eigenvalues. counted according to multiplicity, by

$$
0<\lambda_{1} \leq \lambda_{2} \leq \cdots \leq \lambda_{1} \leq \cdots \rightarrow \infty \text { as } j \rightarrow \infty \text {. }
$$

The problem of determining the geometry of $\Omega$ has been discussed by Pleijel [4], McKean and Singer [3], Waechter [5], Gottlieb [1], Hsu [2] and Zayed [6-8, 11], using the asymptotic expansion of the spectral function

$$
\Theta(t)=\sum_{j=1}^{\infty} \exp \left(-t \lambda_{\jmath}\right) \text { as } t \rightarrow 0 .
$$

It has been shown that, in the case of Dirichlet boundary conditions (D.b.c)

$$
\Theta(t)=\frac{V}{(4 \pi t)^{3 / 2}}-\frac{|S|}{16 \pi t}+\frac{1}{12 \pi^{3 / 2} t^{\frac{1}{2}}} \int_{S} H d S+a_{0}+0\left(t^{\frac{1}{2}}\right) \text { as } t \rightarrow 0,
$$

while, in the case of Neumann boundary conditions (N.b.c.),

$$
\Theta(t)=\frac{V}{(4 \pi t)^{3 / 2}}+\frac{|S|}{16 \pi t}+\frac{1}{12 \pi^{3 / 2} t^{\frac{1}{2}}} \int_{S} H d S+a_{0}+0\left(t^{\frac{1}{2}}\right) \text { as } t \rightarrow 0,
$$

In these formulae, $V$ and $|S|$ are respectively the volume and the surface area of $\Omega$, while 
$H=\frac{1}{2}\left(\frac{1}{R_{1}}+\frac{1}{R_{2}}\right)$ is the mean curvature of $S$, where $R_{1}$ and $R_{2}$ are the principal radii of curvature. Furthermore, the constant term $a_{0}$ in (1.5) and (1.6) has the following forms:

$a_{0}=\left\{\begin{array}{l}\frac{1}{512 \pi} \int_{S}\left(\frac{1}{R_{1}}-\frac{1}{R_{2}}\right)^{2} d S, \text { in the case of D.b.c. (see [5]), } \\ \frac{7}{512 \pi} \int_{S}\left(\frac{1}{R_{1}}-\frac{1}{R_{2}}\right)^{2} d S, \text { in the case of N.b.c. (see [2]). }\end{array}\right.$

In terms of the mean curvature $H$ and Gaussian curvature $N=\frac{1}{R_{1} R_{2}},(1.7)$ may be rewritten in the forms:

$a_{0}=\left\{\begin{array}{l}\frac{1}{128 \pi} \int_{S}\left(H^{2}-N\right) d S, \text { in the case of D.b.c. } \\ \frac{7}{128 \pi} \int_{S}\left(H^{2}-N\right) d S, \text { in the case of N.b.c. }\end{array}\right.$

The object of this paper is to discuss the following more general inverse problem: Let $\Omega$ be an arbitrary multiply connected bounded region in $R^{3}$ which is surrounded internally by simply connected bounded domains $\Omega_{1}$ with smooth bounding surfaces $S_{i}, i=1,2, \ldots, m-1$, and externally by a simply connected bounded domain $\Omega_{m}$ with a smooth bounding surface $S_{m}$. Suppose that the eigenvalues (1.3) are given for the eigenvalue equation

$$
\left(\Delta_{3}+\lambda\right) u=0 \text { in } \Omega,
$$

together with one of the following mixed boundary conditions:

$$
\frac{\partial u}{\partial n_{i}}=0 \text { on } S_{i}, \quad i=1, \ldots, k, \quad u=0 \text { on } S_{z}, \quad i=k+1, \ldots m,
$$

or

$$
u=0 \text { on } S_{v}, i=1, \ldots, k, \frac{\partial u}{\partial n_{i}}=0 \text { on } S_{i}, \quad i=k+1, \ldots, m,
$$

where $\frac{\partial}{\partial n_{i}}$ denote differentiations along the inward pointing normals to $S_{i}, i=1, \ldots, m$. Determine the geometry of $\Omega$ from the asymptotic form of the spectral function $\theta(t)$ for small positive $t$.

Note that problem (1.9)-(1.11) has been investigated recently by Zayed [11] in the special case when $\Omega$ is an arbitrary doubly connected region (i.e., $m=2$ ).

\section{STATEMENT OF RESULTS.}

Suppose that the bounding surfaces $S_{1}(i=1, \ldots, m)$ of the region $\Omega$ are given locally ly infinitely differentiable functions $x^{n}=y^{n}\left(\sigma_{2}\right), n=1,2,3$, of the parameters $\sigma_{1}^{\alpha}=$ constants, are linen of curvature, the first and second fundamental forms of $S_{i}(i=1, \ldots, m)$ can be written respectively. in the following forms:

$$
\prod_{1 i}\left(\sigma_{i}, \Delta \sigma_{\imath}\right)=a_{1 i}\left(\sigma_{i}\right)\left(\Delta \sigma_{i}^{1}\right)^{2}+a_{2 i}\left(\sigma_{i}\right)\left(\Delta \sigma_{i}^{2}\right)^{2}
$$

and

$$
\prod_{2 i}\left(\sigma_{i}, \Delta \sigma_{i}\right)=b_{1 i}\left(\sigma_{i}\right)\left(\Delta \sigma_{i}^{1}\right)^{2}+b_{2 i}\left(\sigma_{i}\right)\left(\Delta \sigma_{i}^{2}\right)^{2}
$$

In terms of the coefficients $a_{1 v}, a_{2 i}, b_{1}, b_{2 i}$ the principal radii of curvatures for $S_{\mathfrak{a}}(i=1, \ldots, m)$ are given by:

$$
R_{1 \mathrm{i}}=a_{1 \mathrm{i}} / b_{1 \mathrm{i}} \text { and } R_{2 \mathrm{i}}=a_{2 \mathrm{i}} / b_{2 \mathrm{i}} \text {. }
$$

Consequently, the mean curvatures $H_{1}$ and Gaussian curvatures $N_{1}$ of the bounding surfaces $S_{i}(i=1, \ldots, m)$ are defined by:

$$
H_{\mathrm{i}}=\frac{1}{2}\left(\frac{1}{R_{1 \mathrm{i}}}+\frac{1}{R_{2 \mathrm{i}}}\right) \text { and } N_{\mathrm{i}}=\frac{1}{R_{1 \mathrm{t}} R_{2 \mathrm{i}}} \text {. }
$$

Let $\left|S_{i}\right|,(i=1, \ldots, m)$ be the surface areas of the bounding surfaces $S_{i}(i=1, \ldots m)$ respectively. Then, the results of problem (1.9)-(1.11) can be summarized in the following cases:

CASE 1. (N.b.c. on $S_{\imath}, i=1, \ldots, k$ and D.b.c. on $S_{\imath}, i=k+1, \ldots, m$ )

$$
\begin{gathered}
\Theta(t)=\frac{V}{(4 \pi t)^{3 / 2}}+\frac{1}{16 \pi t}\left\{\sum_{i=1}^{k}\left|S_{i}\right|-\sum_{i=k+1}^{m}\left|S_{i}\right|\right\}+\frac{1}{12 \pi^{3 / 2} t^{\frac{1}{2}}} \sum_{i=1}^{m} \int_{S_{i}} H_{i} d S_{\mathrm{i}} \\
+\frac{1}{128 \pi}\left\{7 \sum_{i=1}^{k} \int_{S_{i}}\left(H_{i}^{2}-N_{i}\right) d S_{i}+\sum_{i=k+1}^{m} \int_{S_{i}}\left(H_{i}^{2}-N_{i}\right) d S_{i}\right\}
\end{gathered}
$$




$$
\begin{aligned}
& +\left(t / \pi^{3}\right)^{\frac{1}{2}}\left\{\frac{13}{1440} \sum_{i=1}^{k} \int_{S_{1}} H_{i}^{3} d S_{i}-\frac{1}{315} \sum_{i=k+1}^{m} \int_{S_{i}} H_{i}^{3} d S_{i}\right\} \\
& +0(t) \text { as } t \rightarrow 0 .
\end{aligned}
$$

CASE 2. (D.b.c. on $S_{\imath}, i=1, \ldots, k$ and N.b.c. on $S_{v}, i=k+1, \ldots m$ )

In this case, the asymptotic expansion of $\Theta(t)$ as $t \rightarrow 0$ follows directly from (2.1) with the interchanges $S_{1}, i=1, \ldots, k \leftarrow S_{1}, i=k+1, \ldots, m$.

With reference to formulae (1.5), (1.6) and to the articles [1], [2], [7], [11], the asymptotic expansion (2.1) may be interpreted as follows:

(i) $\Omega$ is an arbitrary multiply connected bounded region in $R^{3}$ and we have the mixed boundary conditions (1.10) or (1.11) as indicated in the specifications of the two respective cases.

(ii) For the first five terms, $\Omega$ is an arbitrary multiply connected bounded region in $R^{3}$ of volume $\mathrm{V}$.

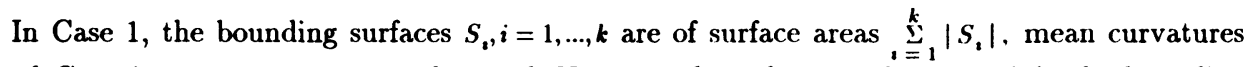
$H_{1}$ and Gaussian curvature $N_{i}$ together with Neumann boundary conditions, while the hounding surfaces $S_{\imath}, i=k+1, \ldots, m$ are of surface areas $\sum_{i=k+1}^{m}\left|S_{\imath}\right|$, mean curvatures $H_{1}$ and Gaussian curvature $N_{z}$ together with Dirichlet boundary conditions.

We close this section with the following remarks:

REMARK 2.1. On setting $k=0$ in (2.1) with the usual definition that $\sum_{i=1}^{0}$ is zero, we obtain the result of D.b.c. on $S_{1}, i=1, \ldots, m$.

REMARK 2.2. On setting $k=m$ in (2.1) with the usual definition that $\underset{i=m+1}{m}$ is zero, we obtain the result of N.b.c. on $S_{i}, i=1, \ldots, m$.

\section{FORMULATION OF THE MATHEMATICAL PROBLEM.}

In analogy with the two-dimensional problem (see $[9,10])$, it is easy to show that $\Theta(t)$ associated with problem (1.9)-(1.11) is given by:

$$
\Theta(t)=\iint_{\Omega} \int(i(\underline{\sim}, \underline{\sim}: t) d \underline{\sim} .
$$

where $G\left({\underset{x}{1}}_{1}, \underline{x}_{2} ; t\right)$ is Green's function for the heat equation

$$
\left(\Delta_{3}-\frac{\partial}{\partial t}\right) u=0 .
$$

subject to the mixed boundary conditions $(1.10)$ or (1.11) and the initial condition

$$
\lim _{t \rightarrow 0} G\left(x_{1}, x_{2} ; t\right)=\delta\left(x_{1}-\underline{x}_{2}\right),
$$

where $\delta\left(x_{1}-x_{2}\right)$ is the Dirac delta function located at the source point, $\underset{\sim}{x} 2$.

Let us write

$$
G\left(\underline{x}_{1}, x_{2} ; t\right)=G_{0}\left(\underline{x}_{1}, \underline{x}_{2} ; t\right)+\chi\left(\underline{x}_{1}, \underline{\sim}_{2} ; t\right)
$$

where

$$
G_{0}\left(x_{1}, x_{2} ; t\right)=(4 \pi t)^{-3 / 2} \exp \left\{-\frac{\left|\underline{x}_{1}-x_{2}\right|^{2}}{4 t}\right\},
$$

is the "fundamental solution" of the heat equation (3.2) while $\chi\left(x_{1}, x_{2} ; t\right)$ is the "regular solution" chosen so that $G\left(x_{1}, \sim_{2} ; t\right)$ satisfies the mixed boundary conditions $(1.10)$ or (1.11).

On setting $\underset{\sim}{x_{1}}={\underset{x}{2}}_{2}=\underset{\sim}{x}$ we find that

$$
\Theta(t)=\frac{V}{(4 \pi t)^{3 / 2}}+K(t) .
$$

where

$$
K(t)=\iint_{\Omega} \int_{\Omega} \lambda(x, x ; t) d x .
$$

In what follows, we shall use Laplace transforms with respect to $t$, and use $s^{2}$ as the Laplace transform parameter; thus we define

$$
\bar{G}\left(x_{1}, x_{2} ; s^{2}\right)=\int_{0}^{+\infty} e^{-s^{2} t}(;(\underbrace{}_{1}, \underbrace{}_{2} ; t) d t .
$$


An application of the Laplace transform to the heat equation (3.2) shows that $\bar{G}\left(\underline{x}_{1}, x_{2} ; s^{2}\right)$ satisfies the membrane equation

$$
\left(\Delta_{3}-s^{2}\right) \bar{G}\left(\underline{\sim}_{1}, \underline{\sim}_{2} ; s^{2}\right)=-\delta\left(x_{1}-x_{2}\right) \text { in } \Omega,
$$

together with the mixed boundary conditions (1.10) or (1.11).

The asymptotic expansion of $K(t)$ as $t \rightarrow 0$, may then be deduced directly from the asymptotic expansion of $\bar{K}\left(s^{2}\right)$ as $s \rightarrow \infty$, where

$$
\bar{K}\left(s^{2}\right)=\iint_{\Omega} \int \bar{x}\left(x, x ; s^{2}\right) d x .
$$

\section{CONSTRUCTION OF GREEN'S FUNCTION.}

It is well known [7] that the membrane equation (3.9) has the fundamental solution

$$
\bar{G}_{0}\left(x_{1}, x_{2} ; s^{2}\right)=\frac{\exp \left(-s r_{x_{1}} x_{2}\right)}{4 \pi r_{x_{1} x_{2}}},
$$

where $r_{x_{1}}, x_{2}=\left|x_{1}-x_{2}\right|$ is the distance between the points $x_{1}=\left(x_{1}^{1}, x_{1}^{2}, x_{1}^{3}\right)$ and $x_{2}=\left(x_{2}^{1}, x_{2}^{2}, x_{2}^{3}\right)$ of the domain $\Omega$. The existence of the solution (4.1) enables us to construct integral equations for $\bar{G}\left(x_{1}, x_{2} ; s^{2}\right)$ satisfying the mixed boundary conditions (1.10) or (1.11). Therefore, in Case 1, Green's theorem gives:

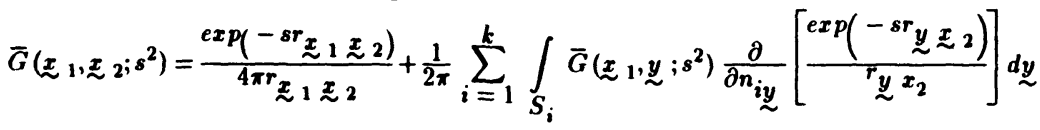

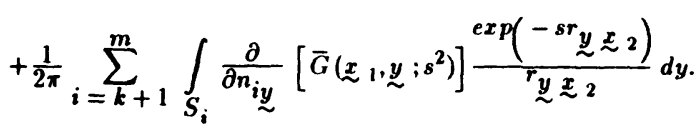

On applying the iteration method (see [7], [9], [11]) to the integral equation (4.2), we obtan the Green's function $\bar{G}\left(x_{1}, x_{2} ; s^{2}\right)$ which has the regular part:

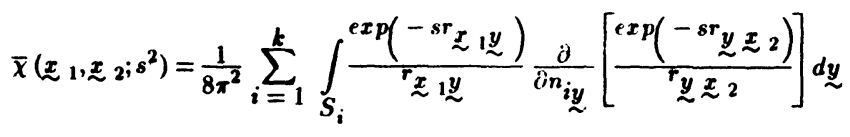

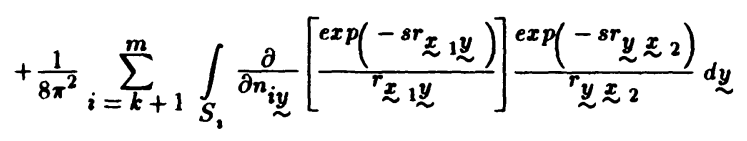

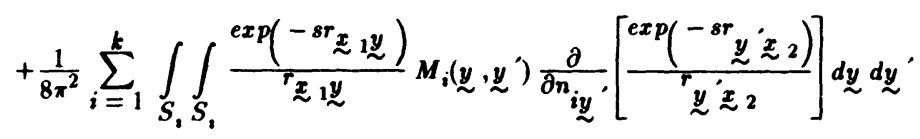

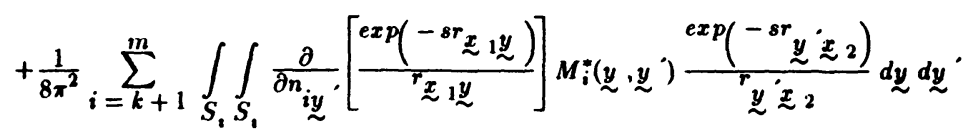

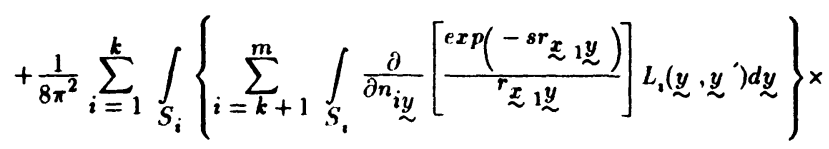

$$
\begin{aligned}
& \times \frac{\partial}{\partial n_{i y}} \cdot\left[\frac{\exp \left(-s r^{\prime} \underline{y}_{2}\right)}{r^{y^{\prime} x_{2}} x_{2}}\right] d \underline{y}
\end{aligned}
$$

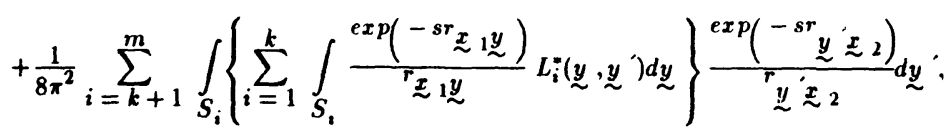


where

$$
\begin{aligned}
& M_{1}\left(\underline{\sim}, \underline{\sim}^{\prime}\right)=\sum_{\nu=0}^{\infty} K_{i}^{(\nu)}\left(\underline{\sim}, y^{\prime}\right) . \\
& M_{i}^{*}\left(\underline{\underline{y}}, \underline{\sim}^{\prime}\right)=\sum_{\nu=0}^{\infty}{ }^{*} K_{i}^{(\nu)}\left(\underline{\sim}^{\prime}, \underline{\sim}\right), \\
& L_{\imath}\left(\underline{\sim}, \underline{\sim}^{\prime}\right)=\sum_{\nu=0}^{\infty} K_{\underline{\sim}}^{(\nu)}\left(\underline{\sim}^{\prime}, \underline{\sim}\right), \\
& L_{i}^{*}\left(\underline{\sim}_{\underline{y}}^{\underline{y}}\right)=\sum_{\nu=0}^{\infty} \boldsymbol{K}^{(\nu)} \cdot(\underset{\sim}{(y}, \underset{\sim}{y}),
\end{aligned}
$$

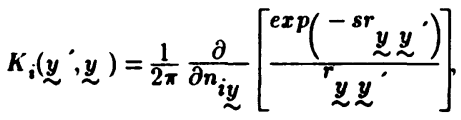

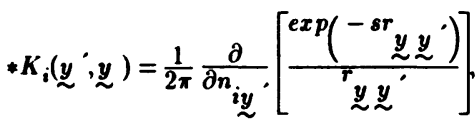

$$
\begin{aligned}
& K_{-1}\left(\underline{y}^{\prime}, \underline{\sim}\right)=\frac{1}{2 \pi} \frac{\exp \left(-s r \underline{y} \underline{w}^{\prime}\right)}{\underline{r}^{y} \underline{\sim}^{\prime}},
\end{aligned}
$$

and

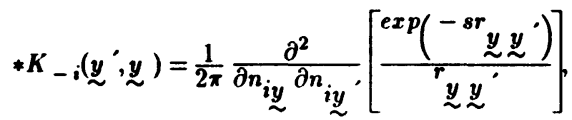

In the same way, we can show that in Case 2, the Green's function $\bar{G}\left(x_{1}, x_{2} ; s^{2}\right)$ has a regular part of the same form (4.3) with the interchanges $S_{2}, z=1, \ldots, k \mapsto S_{2}, i=k+1, \ldots ., m$.

On the basis of (4.3) the function $\bar{\chi}\left(x_{1}, x_{2} ; s^{2}\right)$ will be estimated for $s \rightarrow \infty$. The case when $\underline{\sim}$ and $\underline{x}_{2}$ lie in the neighborhood of the bounding surfaces $S_{i}, i=1, \ldots, m$ of $\Omega$ is particulari: interesting. For this case, we need to use the following coordinates.

\section{COORDINATES IN THE NEIGHBORHOOD OF $S_{i}, i=1, \ldots, m$.}

Let $h_{i}>0(i=1, \ldots, m)$ be sufficiently small. Let $n_{i}(i=1, \ldots, m)$ be the minimum distances from a point $z=\left(x^{1}, x^{2}, x^{3}\right)$ of the domain $\Omega$ to its bounding surfaces $S_{i}(i=1, \ldots, m)$ respectively. Let $n_{i}\left(\sigma_{i}\right)(i=1, \ldots, m)$ denote the inward drawn unit normals to $S_{1}(i=1, \ldots, m)$ respectively. We note that the coordinates in the neighborhood of $S_{i}(i=k+1, \ldots, m)$ are in the same form as in Section 5.1 of [11] with the interchanges $\sigma_{2}^{1} \leftrightarrow \sigma_{i}^{1}, \sigma_{2}^{2} \leftrightarrow \sigma_{i}^{2}, n_{2} \leftrightarrow n_{i}, h_{2} \leftrightarrow h_{i}, I_{2} \leftrightarrow I_{i}, g\left(I_{2}\right) \leftrightarrow g\left(I_{i}\right)$ and $\delta_{2}$ $\leftrightarrow \delta_{1},(i=k+1, \ldots, m)$. Thus we have the same formulae (5.1.1)-(5.1.6) of Section 5.1 in [11] with the interchanges $n_{2} \leftrightarrow n_{i}, \underset{\sim}{n_{2}}\left(\sigma_{2}\right) \leftrightarrow \underset{\sim}{n_{i}}\left(\sigma_{i}\right), I I_{1} \leftrightarrow I I_{1 i}, I I_{2} \leftrightarrow I I_{2 i}, H_{1} \leftrightarrow H_{1}$ and $N_{1} \leftrightarrow N_{1}$ $(i=k+1, \ldots, m)$.

Similarly, the coordinates in the neighborhood of $S_{i},(i=1, \ldots, k)$ are similar to those obtained in Section 5.2 of [11] with the interchanges $\sigma_{1}^{1} \leftrightarrow \sigma_{i}^{1}, \sigma_{1}^{2} \rightarrow \sigma_{1}^{2}, n_{1} \leftrightarrow n_{i}, h_{1} \rightarrow h_{i}, I_{1} \leftrightarrow I_{i}, \Phi\left(I_{1}\right) \leftrightarrow$ $\Phi\left(I_{i}\right)$ and $\delta_{1} \leftrightarrow \delta_{i},(i=1, \ldots, k)$. Thus, we have the same formulae (5.2.1)-(5.2.5) of Section 5.1 in [11] with the interchanges $n_{2} \rightarrow n_{\mathfrak{v}}, \underset{\sim}{n_{2}}\left(\sigma_{2}\right) \rightarrow n_{,}^{n}\left(\sigma_{1}\right), I I_{1}^{*}-I I_{1}, I I_{2}^{*} \rightarrow I I_{2 v}, H_{1}^{*} \rightarrow H_{i}$ and $N_{i}^{*} \rightarrow$ $N_{n},(i=k+1, \cdots m)$

6. SOME LOCAL EXPANSIONS.

It now follows that the local expansions of the functions

$$
\frac{\exp \left(-s r_{\underline{x}} \underset{\sim}{y}\right)}{{ }^{r_{x}} \underset{\sim}{y}}, \frac{\partial}{\partial n_{i y}}\left[\frac{\exp \left(-s r_{\underline{x}} \underset{\sim}{y}\right)}{{ }^{r_{x}} \underset{\sim}{y}}\right], i=1, \ldots, m .
$$


when the distance between $\underset{x}{x}$ and $\underset{\sim}{y}$ is small are very similar to those obtained in Section 6 of [11]. Consequently, the local behavior of the kernels

$$
\begin{aligned}
& K_{,}\left(\underline{\sim}^{\prime}, \underline{\sim}\right),{ }^{*} K_{-8}\left(y_{\sim}^{\prime}, \underset{\sim}{y}\right), \\
& * K_{1}(\underset{\sim}{\prime}, \underset{\sim}{y}), K_{-}(y_{\sim}^{\prime}, \underbrace{y}_{\sim}),
\end{aligned}
$$

when the distance between $\underset{\sim}{y}$ and ${\underset{\sim}{y}}^{\prime}$ is small, follows directly from the local expansions of the functions (6.1).

DEFINITION 1. If ${\underset{\xi}{1}}_{1}$ and ${\underset{\sim}{2}}_{2}$ are points in the half-part $\xi^{3}>0$, then we define

$$
\hat{\rho}_{12}=\sqrt{\left(\xi_{1}^{1}-\xi_{2}^{1}\right)^{2}+\left(\xi_{1}^{2}-\xi_{2}^{2}\right)^{2}+\left(\xi_{1}^{3}+\xi_{2}^{3}\right)^{2}} .
$$

An $e^{\lambda}\left({\underset{\sim}{1}}_{1}, \underline{\sim}_{2} ; s\right)$-function is defined for points ${\underset{\xi}{1}}_{1}$ and $\underline{\xi}_{2}$ belong to sufficiently small domains $\boldsymbol{Y}\left(\boldsymbol{I}_{i}\right)$ except when $\underline{\xi}_{1}={\underset{\xi}{\xi}}_{2} \in I_{i},(i=1, \ldots, m)$ and $\lambda$ is called the degree of this function. For every positive integer $\Lambda$, it has the local expansion (see [11]):

$$
e^{\lambda}\left(\underline{\xi}_{1}, \underline{\sim}_{2} ; s\right)=\Sigma^{*} f\left(\xi_{1}^{1}, \xi_{1}^{2}\right)\left(\xi_{1}^{3}\right)^{P_{1}}\left(\xi_{2}^{3}\right)^{P_{2}}\left(\frac{\partial}{\partial \xi_{1}^{1}}\right)^{\ell_{1}}\left(\frac{\partial}{\partial \xi_{1}^{3}}\right)^{\ell_{2}}\left(\frac{\partial}{\partial \xi_{1}^{3}}\right)^{\ell_{3}} \frac{\exp \left(-s \hat{\rho}_{12}\right)}{\hat{\rho}_{12}}+R^{\wedge}\left(\underline{\sim}_{1}, \underline{\sim}_{2} ; s\right),
$$

where $\Sigma^{*}$ denotes a sum of a finite number of terms in which $f\left(\xi^{1}, \xi^{2}\right)$ are infinitely differentiable functions. In this expansion $P_{1}, P_{2}, l_{1}, l_{2}, l_{3}$ are integers, where $P_{1} \geq 0, P_{2} \geq 0, l_{1} \geq 0, l_{2} \geq 0$. $\lambda=\min \left(P_{1}+P_{2}-q\right), q=\ell_{1}+\ell_{2}+\ell_{3}$ and the minimum is taken over all terms which occur in the summation $\Sigma^{*}$. The remainder $R^{\Lambda}\left(\xi_{1}, \underline{\sim}_{2} ; s\right)$ has continuous derivatives of order $d \leq \Lambda$ satisfying

$$
D^{d} R^{\Lambda}\left(\underset{\sim}{\xi},{\underset{\sim}{\xi}}_{2} ; s\right)=0\left[s^{-\Lambda} \exp \left(-A s \widehat{\rho}_{12}\right)\right] \text { as } s \rightarrow \infty,
$$

where $A$ is a positive constant.

Thus, using methods similar to those obtained in Section 7 of [11], we can show that the functions (6.1) are $e^{\lambda}$-functions with degrees $\lambda=-1,-2$ respectively. Consequently, the functions (6.2) are $e^{\lambda}$-functions with degrees $\lambda=0,-1$ while the functions (6.3) are $e^{\lambda}$-functions with degrees $\lambda=0,1$ respectively.

DEFINITION 2. If ${\underset{\sim}{1}}_{1}$ and ${\underset{\sim}{2}}_{2}$ are points in large domains $\Omega+S_{4}$, then we define

and

$$
\hat{r}_{12}=\min _{\nu}\left(r_{x} \underset{\sim}{y}+r_{x} \underset{\sim}{y}\right) \text { if } \underset{\sim}{y} \in S_{i}, i=1, \ldots, k
$$

$$
\widehat{R}_{12}=\min _{\sim}\left(r_{x_{1}} \underset{\sim}{y}+r_{x_{2}} \underset{\sim}{y}\right) \text { if } \underset{\sim}{y} \in S_{i}, i=k+1, \ldots m .
$$

An $E^{\lambda}\left(x_{1}, x_{2} ; s\right)$-function is defined and infinitely differentiable with respect to $x_{1}$ and $\boldsymbol{x}_{2}$ when these points belong to large domains $\Omega+S_{i}$ except when ${\underset{x}{1}}_{1}=x_{2} \in S_{i}, i=1, \ldots, m$. Thus, the $E^{\lambda}$-function has a similar local expansion of the $e^{\lambda}$-function (see [7], [11]).

With the help of Section 8 in [11], it is easily seen that formula $(4.3)$ is an $E^{-2}\left(x_{1}, x_{2} ; 8\right)$ function and consequently

$$
\bar{G}\left(x_{1}, x_{2} ; s^{2}\right)=\sum_{i=1}^{k}\left\{\left\{\hat{r}_{12}^{-2} \exp \left(-A_{i} s \widehat{r}_{12}\right)\right\}+\sum_{i=k+1}^{m} 0\left\{\widehat{R}_{12}^{-2} \exp \left(-A_{i} s \widehat{R}_{12}\right)\right\},\right.
$$

which is valid for $s \rightarrow \infty$, where $A_{i}(i=1, \ldots, m)$ are positive constants. Formula (6.4) shows that $\vec{G}\left(x_{1}, x_{2}, s^{2}\right)$ is exponentially small for $s \rightarrow \infty$.

With reference to Sections 7 and 9 in [11], if the $e^{\lambda}$-expansions of the functions (6.1)-(6.3) are introduced into (4.3) and if we use formulae similar to (7.4) and (7.10) of Section 7 in [11], we obtain the following local behavior of $\bar{\chi}\left(x_{1}, x_{2} ; s^{2}\right)$ as $s \rightarrow \infty$ which is valid when $\widehat{r}_{12}$ and $\widehat{R}_{12}$ are small: 


$$
\bar{\chi}\left(\underline{\sim}_{1}, \underline{\sim}_{2} ; s^{2}\right)=\sum_{i=1}^{m} \bar{\chi}_{2}\left(\underline{x}_{1}, \underline{x}_{2} ; s^{2}\right),
$$

where, if $\underset{\sim}{x}$ and ${\underset{\sim}{x}}_{2}$ belong to sufficiently small domains $\Phi\left(I_{2}\right), i=1, \ldots, m$, then

$$
\bar{\chi}_{1}\left(x_{1}, x_{2} ; s^{2}\right)=-\frac{\exp \left(-s \hat{\rho}_{12}\right)}{8 \pi \hat{\rho}_{12}}+0\left\{\frac{\exp \left(-A_{1} s \hat{\rho}_{12}\right)}{\hat{\rho}_{12}}\right\} \text { as } s \rightarrow \infty \text {. }
$$

When $\widehat{r}_{12} \geq \delta_{i}>0, i=1, \ldots, k$ and $\widehat{R}_{12} \geq \delta_{1}>0, i=k+1, \ldots, m$, the function $\left.\bar{\gamma}_{\left(x_{1}, x\right.} ; ; s^{2}\right)$ is of order $0\left\{\exp \left(-s N_{0}\right)\right\}$ as $s \rightarrow \infty, N_{0}>0$. Thus, since $\lim _{r_{12} \rightarrow 0} \frac{\widehat{r}_{12}}{\widehat{\rho}_{12}}=\lim _{R_{12} \rightarrow 0} \frac{\widehat{R}_{12}}{\widehat{\rho}_{12}}=1$ (see [11]), then the local behavior of the formula (4.3) has the form (6.5), where if $\underline{x}_{1}$ and ${\underset{\sim}{\tau}}_{2}$ belong to large domains $\Omega+S_{\imath}, i=1, \ldots, k$, we get

$$
\bar{\chi}_{2}(\underbrace{}_{1}, \underbrace{}_{2} ; s^{2})=-\frac{\exp \left(-s \hat{r}_{12}\right)}{8 \pi \widehat{r}_{12}}+0\left\{\frac{\exp \left(-A_{t} s \widehat{r}_{12}\right)}{\widehat{r}_{12}}\right\} \text { as } s \rightarrow \infty,
$$

while, if $\underset{\sim}{x}$ and ${\underset{x}{x}}_{2}$ belong to large domains $\Omega+S_{i}, i=k+1, \ldots, m$, we get:

$$
\bar{\chi}_{1}\left(x_{1}, \underline{\sim}_{2} ; s^{2}\right)=-\frac{\exp \left(-s \widehat{R}_{12}\right)}{8 \pi \widehat{R}_{12}}+\left\{\frac{\exp \left(-A_{i} s \widehat{R}_{12}\right)}{\widehat{R}_{12}}\right\} \text { as } s \rightarrow \infty .
$$

\section{CONSTRUCTION OF RESULTS.}

Since for $\xi^{3} \geq h_{i}>0, i=1, \ldots, m$ the functions $\bar{\chi}_{i}\left(x, x ; s^{2}\right)$ are of orders $0\left(e^{-2 A_{i} s h_{i}}\right)$, the integral over $\Omega$ of the function $\bar{\chi}\left(\underset{x}{x} ; s^{2}\right)$ can be approximated in the following way (see (3.10)):

$$
\begin{aligned}
& \bar{K}\left(s^{2}\right)=\sum_{i=k+1}^{m} \int_{S_{i}} \int_{\xi^{3}=0}^{h_{1}} \bar{\chi}_{i}\left(\underset{\sim}{x}, \underline{\sim}: s^{2}\right)\left\{1-2 \xi^{3} H_{i}+\left(\xi^{3}\right)^{2} N_{i}\right\} d \xi^{3} d S_{i} \\
& -\sum_{i=1}^{k} \int_{S_{\imath}} \int_{\xi^{3}=0}^{h_{i}} \bar{\chi}_{\imath}\left(\underset{\sim}{x}, \underline{\sim} ; s^{2}\right)\left\{1+2 \xi^{3} H_{\imath}+\left(\xi^{3}\right)^{2} N_{\imath}\right\} d \xi^{3} d S_{\imath} \\
& +\sum_{i=1}^{m} 0\left(e^{-2 A_{i} s h_{i}}\right) \text { as } s \rightarrow \infty \text {. }
\end{aligned}
$$

If the $e^{\lambda}$-expansions of $\bar{\chi}_{\imath}\left(x, x ; s^{2}\right)$ are introduced into (7.1) and with the help of formula (10.2) of Section 10 in [11], we deduce after inverting Laplace transforms, that

$$
K(t)=\frac{a_{1}}{t}+\frac{a_{2}}{t^{1 / 2}}+a_{3}+a_{4} t^{1 / 2}+0(t) \text { as } t \rightarrow 0,
$$

where

and

$$
\begin{aligned}
& a_{1}=\frac{1}{16 \pi}\left\{\sum_{i=1}^{k}\left|S_{i}\right|-\sum_{i=k+1}^{m}\left|S_{i}\right|\right\} a_{2}=\frac{1}{12 \pi^{3 / 2}} \sum_{i=1}^{m} \int_{S_{1}} H_{i} d S_{i}, \\
& a_{3}=\frac{1}{128 \pi}\left\{7 \sum_{i=1}^{k} \int_{S_{i}}\left(H_{i}^{2}-N_{i}\right) d S_{i}+\sum_{i=k+1}^{m} \int_{S_{1}}\left(H_{i}^{2}-N_{i}\right) d S_{i}\right\}
\end{aligned}
$$

$$
a_{4}=\frac{1}{\pi^{3 / 2}}\left\{\frac{13}{1440} \sum_{i=1}^{k} \int_{S_{1}} H_{i}^{3} d S_{1}-\frac{1}{315} \sum_{i=k+1}^{m} \int_{S_{1}} H_{i}^{3} d S_{1}\right\}
$$

On inserting (7.2) into (3.6) we arrive at our result (2.1). 


\section{REFERENCES}

1. GOTTLIEB, H.P.W., Eigenvalues of the Laplacian with Neumann boundary conditions, $\underline{\mathbf{J}}$. Austral. Math. Soc. Ser. B (1985), 293-309.

2. HSU, P., On the $\Theta$-function of a compact Riemannian manifold with boundary, C.R. Acad. Sci. Ser. I, 309, Paris (1989), 507-510.

3. MCKEAN JR., H.P. \& SINGER, I.M., Curvature and the eigenvalues of the Laplacian, J. Diff. Geom. 1, (1967), 43-69.

4. PLEIJEL, A., On Green's functions and the eigenvalue distribution of the three-dimensional membrane equation, Skandinav. Mat. Konger, XII (1954), 222-240.

5. WAECHTER, R.T., On hearing the shape of a drum: An extension to higher dimensions, Proc. Camb. Philos. Soc. 72 (1972), 439-447.

6. ZAYED, E.M.E., Eigenvalues of the Laplacian: An extension to higher dimensions, IMA. J. Applied Math. 33 (1984), 83-99.

7. ZAYED, E.M.E., An inverse eigenvalue problem for a general convex domain: An extension to higher dimensions, J. Math. Anal. Appl. 112 (1985), 455-470.

8. ZAYED, E.M.E., Eigenvalues of the Laplacian for the third boundary value problem: An extension to higher dimensions, J. Math. Anal. Appl. 130 (1988), 78-96.

9. ZAYED, E.M.E., Heat equation for an arbitrary doubly-connected region in $R^{2}$ with mixed boundary conditions, Z. Angew. Math. Phys. 40 (1989), 339-355.

10. ZAYED, E.M.E., An inverse eigenvalue problem for an arbitrary multiply connected bounded region in $R^{2}$, Internat. J. Math. Math. Sci. 14 (1991), 571-580.

11. ZAYED, E.M.E., Hearing the shape of a general doubly-connected domain in $R^{3}$ with mixed boundary conditions, Z. Angew. Math. Phys. 42 (1991), 547-564.

Permanent Address: Professor E.M.E. Zayed, Mathematics Department, Faculty of Science, Zagazig University, Zagazig, Egypt. 


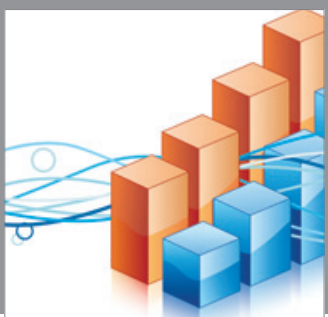

Advances in

Operations Research

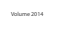

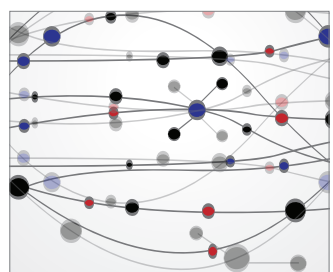

\section{The Scientific} World Journal
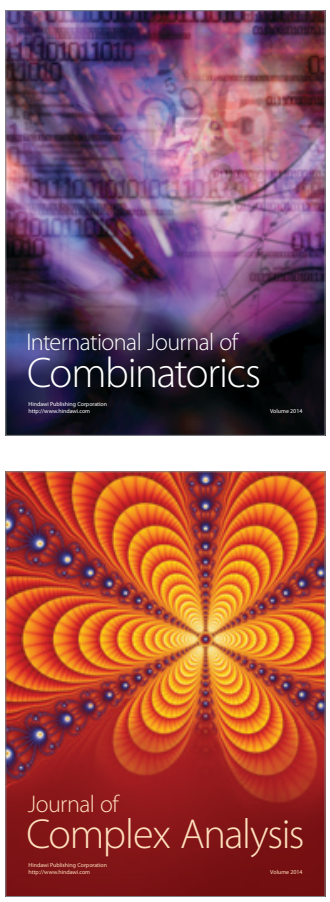

International Journal of

Mathematics and

Mathematical

Sciences
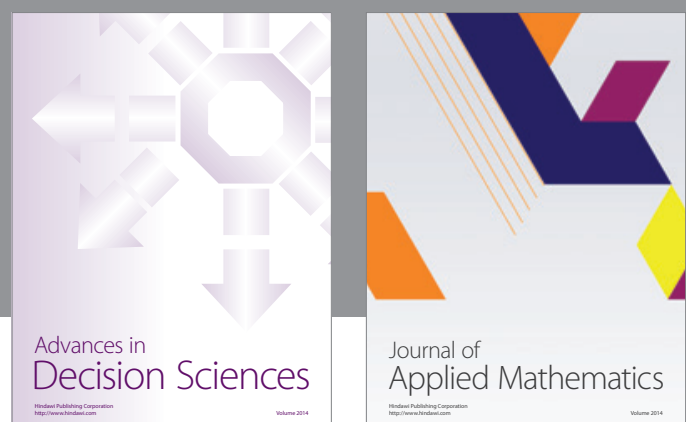

Journal of

Applied Mathematics
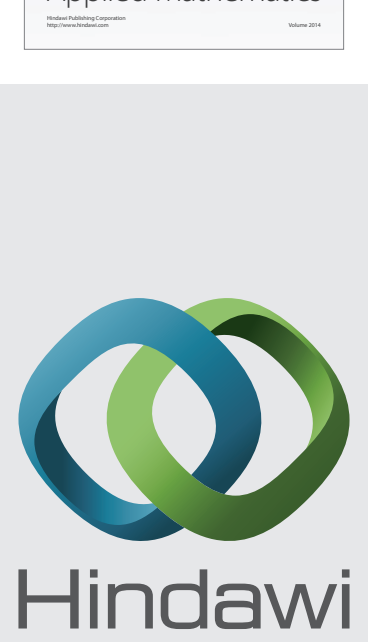

Submit your manuscripts at http://www.hindawi.com
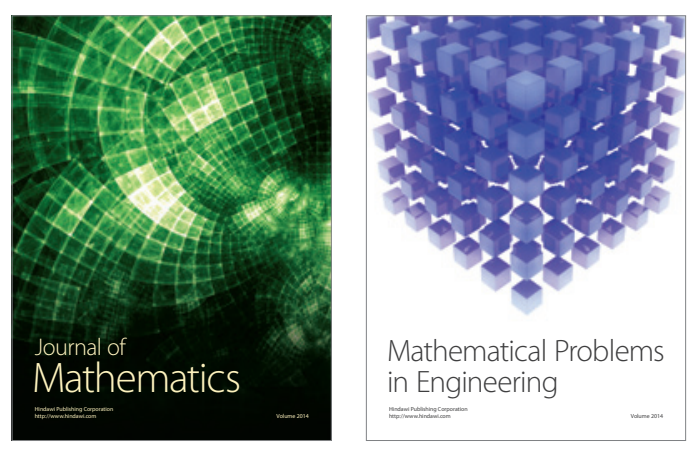

Mathematical Problems in Engineering
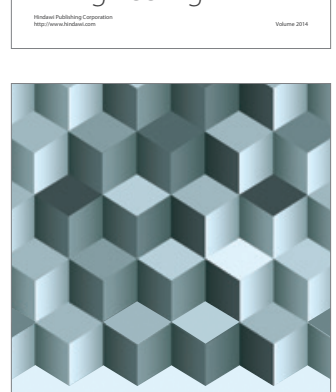

Journal of

Function Spaces
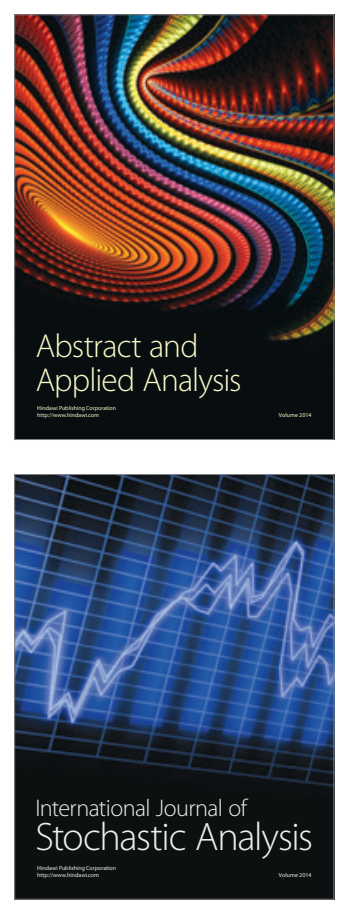

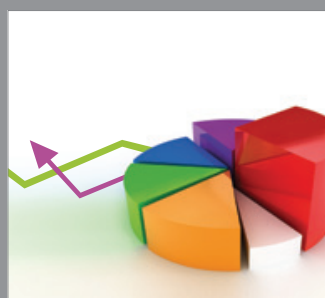

ournal of

Probability and Statistics

Promensencen
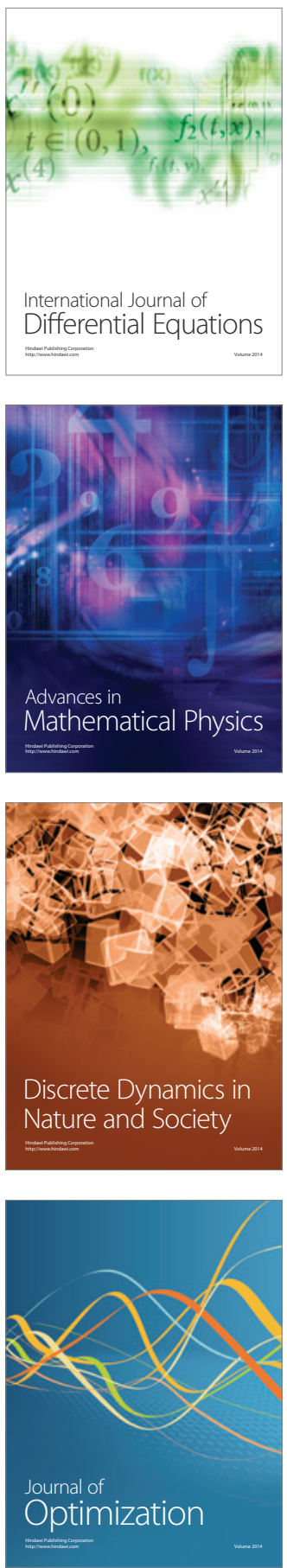